\title{
A Proposed Fuzzy Model for Diseases Diagnosis
}

\author{
Eman Nabil Alkholy, Amal Elsayed Aboutabl, Mohamed Hassan Haggag
}

\begin{abstract}
Decision making has become a problem in environments full of uncertain, vague and imprecise information. They face many problems to train computer systems to simulate human thinking to make the right decision. Different methodologies and approaches have been used to train computers to understand and mimic human thinking. This paper proposes a fuzzy model for a bone disease to have the right diagnosis answer, as a human expertise doctor. and to prove that using fuzzy logic has a significant ability to mimic human thinking. The model accepts inputs in the different forms as physiological and clinical parameters and all data based on medical expertise, using a rule-based fuzzy system approach applied with fourteen rules to have final accurate output decisions. it has been tested in the orthopedic unit against the real existing diagnosis answer from expertise doctor and found that is capable of assisting medical experts in diagnosing diseases and provide good health services to their patients.
\end{abstract}

Keywords: Fuzzy logic, medical Diagnosis, Artificial Intelligence

\section{INTRODUCTION}

$F_{\text {uzzy logic one of artificial intelligent technology that }}$ has seen as one of the most successful technologies in understanding the human brain and deal with sophisticated control and reasoning/inference systems. It depends on the fact of the simulate human brain to solve problems and decision making and generate an accurate answer, and it uses sub-symbolic information to the given model and solution [1]. compared to classic approaches it deduces a good solution from information, by reducing the complexity tremendously. Any gaps in understanding or ambiguous are solved and filled by fuzzy logic, it can accommodate the ambiguities of the real world more aptly due to the formation of soft or vague boundaries, instead of hard (crisp) boundaries. The importance of fuzzy logic systems is the simplicity of operating, in contrary to many other mathematical models. fuzzy logic can solve different problems, Such as sources of subjective information, like expert knowledge and subjective evaluations; (e.g. time series, spatial data), and the presence of different types of data (e.g. quantitative and qualitative data), and the uncertainty of those data [2]. In view of these factors find that fuzzy logic is providing a good solution to various data analytics problems. Furthermore, fuzzy approaches can offer a linguistic concept with excellent approximation in medical care for symptomatic diagnostic remedies.

\section{Revised Manuscript Received on February 05, 2020.}

* Correspondence Author

Eman Nabil Alkholy*, Department of Computer Science and Engineering, Helwan University, Egypt. E-mail: eme.ysaied@gmail.com. prof. Amal Elsayed Aboutabl, Department of Computer Science and Engineering, Helwan University, Egypt. E-mail:aaboutabl@yahoo.com.

Prof. Mohamed Hassan Haggag, Department of Computer Science and Engineering, Helwan University, Egypt. E-mail: mohamed.haggag@fci.helwan.edu.eg.

(C) The Authors. Published by Blue Eyes Intelligence Engineering and Sciences Publication (BEIESP). This is an open access article under the CC BY-NC-ND license (http://creativecommons.org/licenses/by-nc-nd/4.0/)
The aim of this paper is to understand the effect of using fuzzy logic in solving the uncertainty and ambiguous in the diagnosis system, that can be used to design a specific fuzzy expert system for the particular medical domain. An example of a bone disease diagnosis is illustrated in support to describe the concept confined to a closed domain. The vagueness, imprecision can be exemplified with the mechanism of understanding and what is the desired information required by the user. This study will prove to be an effective study in using fuzzy logic and will engender the interest of academicians, researchers to focus more on applied fuzzy logic in different aspects. our believe is to mimic human thinking and understanding to deal with more accuracy in information.

\section{RELATED WORK.}

Owing a vast amount of information readily lead to having a significant increase in the number of data, Fuzzylogic theory, in general, has been shown to be a useful approach for solving ambiguous problems and uses in a different aspect. in a previous study, there are various application systems for using fuzzy logic in health care. [3].Medical modeling in many cases deals with uncertainty and depend on many factors that are unmeasured that lead to low accuracy in diagnosis, in the first research was deal with uncertainty and solve a different problem which was introduced by Zaheh as a methodology for computing with words in order to handle uncertainty[4].

Fuzzy applications have a virtually exploded, affecting things we use every day, they use fuzzy sets to construct fuzzy query languages for purposes of information retrieval [5], and other researchers use linguistic hedge such as very, more or less, much, essentially, slightly called a hedge operator [6][7]. this intelligent gap can understand by computer systems if they use Fuzzy logic in a better way by handling Uncertainty. Fuzzy logic is a generalization of bivalent logic with more flexibility for handling vagueness and ambiguity in early 80 They use the Test-ScoreSemantics act to represent the meaning of dispositions.[8].

It has the ability to evaluate the recognized word if it semantically appropriate according to recognized sentence, and it can predict the possible missing words given a correctly recognized word by applying fuzzy inferencing with the understanding of the presence of ambiguity and uncertainty [9]. and other researchers proposed a fuzzy as a Formal of Concept Analysis (FFCA) approach for conceptual clustering for automatic generation of concept hierarchy from uncertainty information. the first paper reveals the interdisciplinary methodological interdependence between the programming and the linguistic data set and the design/presentation of fully fuzzified electronic language data sets based on the combined principle of Neuro-Fuzzy systems [10] [11]. 
Fuzzy logic and fuzzy reasoning were used to deal with uncertain information for reasoning with Sanskrit grammar, and provide answers to questions using a method that offers word definitions, with the aim of reducing the time lag that results from askers having to wait for answers to a question from various users [12]. In this context, fuzzy logic was used to aggregate the ratings and provide ranked answers in return some of then use a novel method for Information Extraction (IE) that manage any set of knowledge where information is abundant, vague or imprecise by design an intelligent agent and use a Fuzzy Logic as based engine, and because of its flexibility, it can manage sets of accumulated knowledge. Some later research is based on retrieving a collection of documents based on Ontology Similarity by using a Fuzzy Co-clustering algorithm [13].in the same year 2014 they discuss multi-level human emotions recognition method was also proposed using a fuzzy model which was found to detect more emotions with significant accuracy [14][15]. They found that by using a fuzzy model it helps to detect more emotions with significant accuracy, it shows the similarity between the human brain and computer system in the detection of emotions.

\section{PROPOSED FUZZY MODEL FOR DISEASES DIAGNOSIS}

Our main goal is to study the effect of using fuzzy logic on the health care sector and help to eliminate the error and gap with having accurate results. The fuzzy model of Bone Diseases has been applied on a closed domain, that imitates the mechanism of the human brain in thinking. It has the ability to map the real meaning to truth values and solve the vague and imprecise notions that may happen.

The fuzzy model designed with a define particular set of parameters that play a role in Diagnosis for each of the entities that represent patient data and The model accepts physiological inputs specific to the patients, it contains 9 input variables (The input variables considered are (PSA, Pain, Neoplasmatic History, Fever, Local Erithrotis, Antiflame Reaction Joints Pain, Bone Density Measure) and more than 40 Diagnosis Rule. A fuzzy set is created to describe linguistic variables The linguistic variable "Pain", for instance, may have categories (members) of and Membership values are assigned to the linguistic variables such as("Medium", "High", "Very High"," Continuous"," Defused, "Located"), Membership function is then developed for each member in the set. Fuzzy sets were derived by

generalizing the concept of a set that contains various elements. It was defined as $(x \in A)$ and that means if we have an element $x$ it will belonging to a set $A$ or it can be noted as ( $\mathrm{x} \notin \mathrm{A}$ ) it means that an element $\mathrm{x}$ is not a member in $\mathrm{A}$.

The symptoms of the Disease are expressed by the deviation of the observations from their normal values and The patient data is retrieved by matching the symptoms and their severity against the antecedent part of fuzzy to make the right decision as the disease name, The fuzzy decision value is defuzzied by the defuzzification component of the designed model to finally arrive at a crisp decision for the disease diagnosis. All input variables and their membership function ranges are presented in (Table- I). And the the architecture of the proposed fuzzy model consists of three main phases as shown in (Fig .1.).

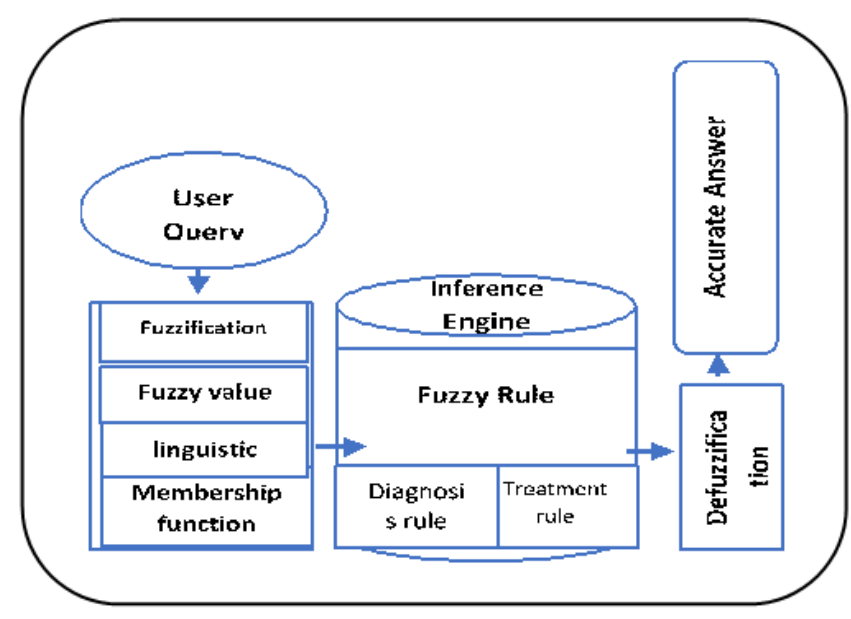

Fig .1. Proposed model

\section{A. FRIST PHASE FUZZIFICATION}

it uses to convert the crisp input values into fuzzy value by giving them membership $\mu \mathrm{A}(\mathrm{x})$ degrees (The grades of membership may be numbered in The interval $[0,1])$ it translates it into linguistic variables represented by fuzzy sets. Then it applies membership functions to the measurements and determines the degree of truth for each rule antecedent.

\section{B. SECOND PHASE INFERENCE}

It has all fuzzy rule is characterized in the form of ifthen rules, as : (If $(\mathbf{x})$ is $\mathbf{A} 1$ and $(\mathbf{y})$ is B1 then $\mathbf{f i}=\mathbf{d i}(\mathbf{x})+\mathbf{s i}(\mathbf{y})$ Where $\mathrm{A} 1$ and $\mathrm{B} 1$ are demonstrated fuzzy sets in the input variables (x), (y); this stage contains diagnosis and treatment rules and it is responsible for all outputs that will be aggregated to form the combined fuzzy output. The rules consist of antecedent and consequent parts. All the rules fire to some extent in the antecedent part of the fuzzy logic system.

\section{THIRD PHASE DEFUZZIFICATION}

the fuzzy output is mapped to a crisp value and the responsible for implanting a nonlinear mapping from its input space to output space through a number of fuzzy 'ifthen' rules is fuzzy inference system.

\section{FORMULATION OF THE RULE BASE}

This model contains 9 input variables and we have created fuzzy set to describe these linguistic variables and every linguistic variable, may have categories (members) of and The Membership function that use as curve that defines how each point in the input space is mapped to a membership value between 0 and 1 , The range between 0 and 1 is referred to as the degree of membership, it allows us to graphically represent a fuzzy set it can be defined as $\mu \mathrm{A}: \mathrm{X} \rightarrow[0,1][16]$. where each element of $\mathrm{X}$ is mapped to a value between ( 0 and 1 ).

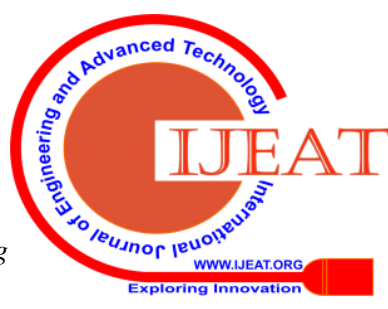


The membership function of $\mathrm{x}$ shows the degree of its membership to a fuzzy set it uses the input values as weighting factors by rules to determine the final output conclusion. it illustrates in (Table- I) upon our empirical data the membership functions of the 9 variables, and the ranges for each input value with the membership function of inputs variables are given in the table

Table- I: Membership functions of input

\begin{tabular}{|c|c|c|c|c|c|c|c|c|c|}
\hline & \multicolumn{9}{|c|}{ Input value } \\
\hline $\begin{array}{c}\text { Membership } \\
\text { Function-MF }\end{array}$ & Pain & $\begin{array}{c}\text { Neoplasmatic } \\
\text { History }\end{array}$ & Fever & $\begin{array}{c}\text { Local } \\
\text { Erithrotis }\end{array}$ & $\begin{array}{c}\text { Antiflame } \\
\text { Reaction }\end{array}$ & $\begin{array}{c}\text { Joints } \\
\text { Pain }\end{array}$ & $\begin{array}{c}\text { Injury } \\
\text { History }\end{array}$ & PSA & $\begin{array}{c}\text { Bone } \\
\text { Density } \\
\text { Measure }\end{array}$ \\
\hline LOW & $1-3.5$ & - & - & - & $1-2.6$ & - & - & - & - \\
\hline HIGH & $10-J u n$ & - & $38.2-40$ & - & $5.6-9.8$ & - & - & - & - \\
\hline CONTINUOUS & $>10$ & - & - & - & - & - & - & - & - \\
\hline MEDIUM & $3.6-5.9$ & - & $37.5-38.2$ & - & $3.6-5.1$ & - & - & - & - \\
& & & & & & & & & \\
\hline DEFUSED & - & - & - & - & - & - & - & - & - \\
\hline LOCATED & - & - & - & - & - & - & - & - & - \\
& & & & & & & & & \\
\hline YES & - & 1 & - & 1 & - & 1 & 1 & 1 & 1 \\
\hline NO & 0.5 & 0.7 & 0 & 0.4 & 0 & 0.7 & 0.6 & 0.5 & 0.6 \\
\hline NORMAL & - & - & $36-37$ & - & - & - & - & - & - \\
\hline
\end{tabular}

\section{EXPERIMENTAL WORK}

The proposed model has been implemented using Fuzzy CLIPS 6.1 expert system. ( Table- II ) shows the membership functions for one of fuzzy sets linguistic. it has one example of membership function for (Antiflame Reaction Input) that his parameter range is divided into four groups ('low', 'medium', 'high' and 'very high'). And also, it shows the range of values and respective fuzzy sets of Antiflame Reaction, Antiflame Reaction is one of the most significant parameters in bone disease.

Table- II: Antiflame Reaction input

\begin{tabular}{|c|c|}
\hline $\begin{array}{c}\text { Range of values and fuzzy sets of Antiflame } \\
\text { Reaction Input }\end{array}$ & $\begin{array}{c}\text { Fuzzy } \\
\text { sets }\end{array}$ \\
\hline $1-2.6$ & Low \\
\hline $3.6-5$ & Medium \\
\hline $5.6-9.8$ & High \\
\hline$>10$ & $\begin{array}{c}\text { Very } \\
\text { High }\end{array}$ \\
\hline
\end{tabular}

\section{IMPLEMENTED FUZZY RULES}

Rule base As previously mentioned in (Table - I), we specified a set of input variables and output variables and give membership function for each parameter that plays a role in diagnosis for each of the entities in the process model that represent patient data and here are a few examples for Diagnosis rule and in (Fig.2.) we present a sample of this fuzzy rules that implemented with Fuzzy Clips.

The following is a sample of fuzzy rules
- Rule 1: If (pain is low) and (fever is NORMAL) and (antinflam-Reaction is low) and (Neoplasmatic-History is no) and (Joints_Pain is no) and (Injury-History is no) and (PSA is no) and (Bone-Density-Measure is no) then (inflammation is no)(chronic-inflammation is no)(primary-benign is no)(special-inflammation is no)(arthritis is no)(local-arthritis is no)(primary-benign is no)(special-inflammation is no)(hard-inflammation is no) (1)

- Rule 2: If (pain is high) and (fever is high) and (antinflam-Reaction is MEDIUM) and (NeoplasmaticHistory is yes) and (Joints_Pain is yes) and (BoneDensity-Measure is yes) then (chronic-inflammation is yes) (1)

- Rule 3: If (pain is CONTINUOUS) and (fever is MEDIUM) and (antinflam-Reaction is MEDIUM) and (Neoplasmatic-History is yes) and (Joints_Pain is yes) and (Bone-Density-Measure is yes) then (inflammation is yes) (1)

- Rule 4: If (Joints_Pain is yes) and (Bone-DensityMeasure is yes) then (inflammation is yes (1).

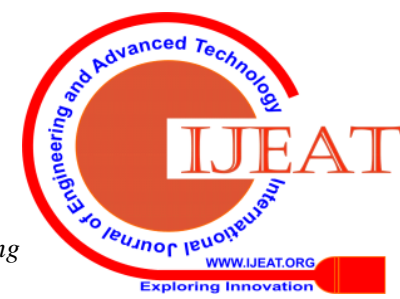




\section{A Proposed Fuzzy Model for Diseases Diagnosis}

Rule: if (pain is contimuous) and (fever is medium) and (antinflam-reaction is medium) and (neoplasmatic-history is yes) and (joints, pain is yes) and (bone-density-measure is yes) then (inflammation is yes) (1)

has been implemented in CLIS P as follows:

(defrule diagnosis_inflammation (possible_diagnosis (disfunction n) (pain continuous)
(fever medium)
(antinflam-reaction medium)
(neoplasmatic-history yes)
(joints pain yes)
(bone-density-measure yes))

$\Rightarrow$ (printout $\mathrm{n}$ "inflammation" crlf)

where the following fact template is used: (deftemplate possible diagnosis

(slot disfunction (type diagnosis))

(slot pain (type fuzzy-value fz-pain))

(slot fever (type fuzzy-value fz- fever ))

(slot antinflam-reaction (type fuzzy-value fz-

antinflam-reaction ))

(slot neoplasmatic-history (type fuzzy-value fzneoplasmatic-history ))

(slot joints_pain (type fuzzy-value fz-joints_pain))

(slot bone-density-measure (type FUZZY-VALUE fz- Bone-Density-Measure ) - Bone-Density-Measure )

Fig2. Sample code for implemented Diagnosis rule

\section{RESULTS}

The proposed model was run for 70 real anonymous patient cases with 40 fuzzy rules to have accurate output depending on the symptom that given as linguistic input after that output results were compared to the results of the expertise doctor and physician in Orthopedic Unit". And we find the accuracy Average 86.56\%, shown in Fig.3. according to the final result, it approved the effectiveness of applying fuzzy methods in the Diseases Diagnosis process, as can be easily seen from the (Table-III), the results of the proposed model for each stage of the reasoning process is quite closer to the expert's real result.

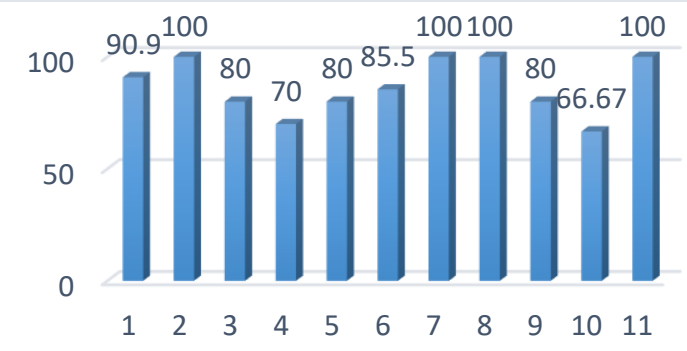

Fig. 3. Proposed Model Accuracy

\section{a. ACCURACY AND ERRor For PROPOSED MODEL}

Accuracy and Error for our proposed model, we used accuracy measure to predict the percentage of right diagnosis comparing with real expert doctor result diagnosis .it used to measure how is our results from the proposed model are near or close to real results from an expert doctor. as shown in equation (1), (NPE) is the number of patients that diagnoses by the expert doctor and (NPD) is the number of patients that diagnoses by the proposed model

$$
A C C U R A C Y=\frac{N P E}{N P D} \times 100 \%
$$

Meanwhile, the Error percentage has calculated the difference or gap between the real expert's results and the predicate result of the proposed model result are multiplied by 100 . As shown in given as equation (2), were (NPE) is the number of patients that diagnoses by the expert doctor and

(NPD) is the number of patients that diagnoses by the proposed model

$$
\text { Erro } \%=\frac{\mathrm{NPD}-\mathrm{NPE}}{\mathrm{TP}} \times 100 \%
$$

Table-III: Comparison Of The Proposed Fuzzy Medical Model Result And The Real Result

\begin{tabular}{|c|c|c|c|c|c|}
\hline $\begin{array}{c}\text { Possible } \\
\text { Diagnosis }\end{array}$ & $\begin{array}{c}\text { Number of } \\
\text { Patients }\end{array}$ & $\begin{array}{c}\text { No. of positive diagnoses by the } \\
\text { proposed model }\end{array}$ & $\begin{array}{c}\text { No. of positive diagnoses by } \\
\text { the expert doctor }\end{array}$ & $\begin{array}{c}\text { Experimental error } \\
\text { number\% }\end{array}$ & $\begin{array}{c}\text { Accuracy\% } \\
\text { primary- } \\
\text { malignant }\end{array}$ \\
\hline
\end{tabular}


2

0

0 100 Applications of Computer Engineering (ACE '13), Cambridge, MA, USA, 30 Jan. - 1 Feb. 2014 ISBN: 978-1-61804-156-2, Pages 77-82

\section{CONCLUSION}

In This paper we have created an intelligent medical model to interact with patients similar to a healthcare support person that would interact with patients to have a right diagnosis upon patient symptoms, it present the effect of using fuzzy logic in the health care sector, The architecture of model has been proposed using Fuzzy Clips 6.5. And it has been tested and implemented successfully, constantly after implementing and measure the accuracy we found that it correctly identifying the right diagnosis answer with $86.56 \%$ accuracy. upon this factor, we found that the model is very effective for the diagnosis of diseases compares with the real result, the model accepts inputs in the form of physiological and clinical parameters and all data based on medical expertise. In aspects of medical experts, they found the model is useful and helpful in diagnosing more diseases and has the ability to provide good health services to their patients. Moreover, it will engender the interest of academicians, researchers to focus more on applied fuzzy logic in different aspects of medical disciplines that are being neglected.

\section{REFERENCES}

1. Zadeh, L. A. (1994). Soft Computing. IEEE Software,11(6),48-56. DOI:10.1109/52.329401

2. Salski, A. (1999) 'Fuzzy Logic Approach to Data Analysis and Ecological Modelling', Proceeding of European Symposium on Intelligent Techniques' (EUFIT99)Orthodox Academy of Crete, Greece.

3. Gürsel G. ( 2016); Healthcare, uncertainty, and fuzzy logic. Digit Med,2:101-12. DOI: 10.4103/2226-8561.194697

4. Zadeh, L. A (1996). Fuzzy Logic Computing With Word IEEE Trans. Fuzzy system 4(2)(1996) 103-111.

5. O. Alhabashneh, R. Iqbal, F. Doctor, and S. Amin. ( 2015), "Adaptive information retrieval system based on fuzzy profiling," 2015 IEEE International Conference on Fuzzy Systems (FUZZ-IEEE), Istanbul, pp. 1-8.

6. Brito A., Barros L., Laureano E., Bertato F., Coniglio M. (2018)," Fuzzy Formal Concept Analysis. In: Barreto G., Coelho R. (eds) Fuzzy Information Processing. NAFIPS ..

7. Tectogrammatical trees and fuzzy natural logic in the linguistic characterization of dynamic processes. ( Conference: 2017 Joint 17th World Congress of International Fuzzy Systems Association and 9th International Conference on Soft Computing and Intelligent Systems (IFSA-SCIS) )June 2017.

8. Zadeh, L. A. (1982). Test-Score Semantics For Natural

9. David Bonanno, Kristen Nock, Leslie Smith, Paul Elmore, and Fred Petry. (2017), "An approach to explainable deep learning using fuzzy inference", Proc. SPIE 10207, Next-Generation Analyst V, 102070D; https://doi.org/10.1117/12.2268001

10. Brito A., Barros L., Laureano E., Bertato F., Coniglio M. (2018)," Fuzzy Formal Concept Analysis. In: Barreto G., Coelho R. (eds) Fuzzy Information Processing. NAFIPS .. Communications in Computer and Information Science, vol 831. Springer, Cham.

11. L. Shen and D. Zhang. (2013), "Formal concept analysis on fuzzy sets," Joint IFSA World Congress and NAFIPS Annual Meeting (IFSA/NAFIPS), Edmonton, AB, 2013, pp. 215-219.

12. Jeremias Leda. (2014)," Fuzzy Controller For Reducing Oscillation On Large Power System, International Journal of Engineering Research \& Technology (IJERT)Vol. 2 Issue 1, January 2014- 2013ISSN: 22780181

13. Rani M., Muyeba M.K., Vyas O.P. (2014) “A Hybrid Approach Using Ontology Similarity and Fuzzy Logic for Semantic Question Answering". In: Kumar Kundu M., Mohapatra D., Konar A., Chakraborty A. (eds) Advanced Computing, Networking, and Informatics- Volume 1. Smart Innovation, Systems and Technologies, vol 27. Springer, Cham

14. Kaveh Bakhtiyari, Hafizah Husain. (2014)," Fuzzy Model on Human Emotions Recognition",12th WSEAS International Conference on
15. Y. Zhang et al. (2016), "Facial Emotion Recognition Based on Biorthogonal Wavelet Entropy, Fuzzy Support Vector Machine, and Stratified Cross-Validation," in IEEE Access, vol. 4, pp. 8375-8385.

16. Sadollah, A. (2018). Introductory Chapter: Which Membership Function is Appropriate in Fuzzy System.

\section{AUTHORS PROFILE}

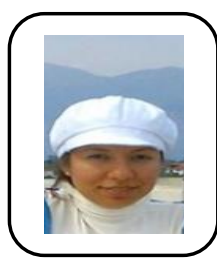

Eman Nabil Alkholy, Currently Pursuing a Ph.D. in Computer Science from Faculty of Computers and Artificial Intelligence, Helwan University, Cairo, Egypt, works as Assistant Lecturer in HOIT. Her research interest includes Fuzzy logic, NLP, Database, vague logic, theoretical Computer Science .and their

applications in Engineering and Healthcare. Which applied in this work. E-mail: eme.ysaied@gmail.com.

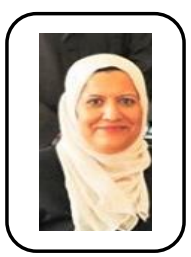

Prof. Amal Elsayed Aboutabl Professor in Department of Computer Science, from Faculty of Computers and Artificial Intelligence, Helwan University, Cairo, Egypt, she was Author and co-author of more than 30 scientific and educational papers. she has more than 15 years of teaching experience at the collegiate level. .Her research interest includes Parallel Processing, Software Engineering, Natural Language Processing. E-mail: aaboutabl@yahoo.com.

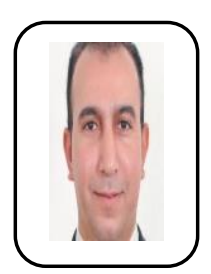

Prof. Mohamed Hassan Haggag Professor in Department of Computer Science, from Faculty of Computers and Artificial Intelligence, Helwan University He has worked as Vice Dean for Research and Postgrad Studies. He has more than 22 years of teaching experience at the collegiate level. He was the author and co-author of more than 150 scientific and educational paper His research areas are Data Mining, Artificial Intelligence, Natural Language Processing. Email:mohamed.haggag@fci.helwan.edu.eg. 